\title{
TOPOLOGICAL SEMIGROUPS WITH INVARIANT MEANS IN THE CONVEX HULL OF MULTIPLICATIVE MEANS( ${ }^{(1)}$
}

\author{
BY \\ ANTHONY TO-MING LAU
}

1. Introduction. Let $S$ be a topological semigroup (i.e. a semigroup with a hausdorff topology such that for each $a \in S$, the mappings $s \rightarrow a s$ and $s \rightarrow s a$, $s \in S$ are continuous from $S$ into $S$ ); let $m(S)[C(S)]$ be the space of bounded [bounded continuous] real functions on $S$. If $f \in m(S), a \in S$, denote by $\|f\|$ $=\sup _{s \in S}|f(s)|$ (sup norm), $p_{a}(f)=f(a), l_{a} f(s)={ }_{a} f(s)=f(a s), r_{a} f(s)=f_{a}(s)=f(s a)$ for $s \in S$, then $p_{a}$ is the point measure on $m(S)$ at $a$ and each element in $\operatorname{Co}\left\{p_{a} ; a \in S\right\}$ is called a finite mean on $m(S)$ (where Co $A$ denotes the convex hull of a subset $A$ of a linear space).

Let $X$ be a translation invariant normed closed subalgebra of $m(S)$ containing constants (i.e. ${ }_{a} f, f_{a} \in X$ whenever $\left.f \in X, a \in S\right), X^{*}$ the conjugate space of $X$ and $L_{a}: X^{*} \rightarrow X^{*}$ such that $\left(L_{a} \varphi\right)(f)=\varphi\left({ }_{a} f\right)$ for $\varphi \in X^{*}, a \in S$ and $f \in X$. An element $\varphi$ in $X^{*}$ is a mean if $\varphi\left(1_{S}\right)=1$ and $\varphi(f) \geqq 0$ for $f \geqq 0\left(1_{S}\right.$ is the constant one function on $S) ; \varphi$ is multiplicative if $\varphi(f g)=\varphi(f) \varphi(g)$ for all $f, g \in X ; \varphi$ is a left invariant mean (LIM) on $X$ if $\varphi$ is a mean and $L_{s} \varphi=\varphi$ for all $s \in S$. A function $f \in C(S)$ is left uniformly continuous if whenever $s_{\alpha} \rightarrow s_{0}, s_{\alpha}, s_{0} \in S,\left\|_{s_{\alpha}} f-{ }_{s_{0}} f\right\| \rightarrow 0$. Denote by LUC $(S)$ the space of left uniformly continuous functions on $S$ (Namioka [17, p. 64]), $\Delta(S)$ the set of multiplicative means on $\operatorname{LUC}(S)$.

The main purpose of this paper is to study and characterize topological semigroups $S$ such that $\left(^{*}\right) \operatorname{LUC}(S)$ has a LIM in Co $\Delta(S)$. We show in $\$ 4$ that any such semigroup can be decomposed as finite disjoint union of open and closed subsets of $S$, one of which is a subsemigroup, $T$, of $S$. LUC $(T)$ contains a subalgebra, $\operatorname{PLUC}(S)$, which admits a multiplicative LIM and the set of LIM on PLUC $(S)$ can be mapped one-one onto the set of LIM on $\operatorname{LUC}(S)$ by a linear transformation. Furthermore, we show that a topological semigroup $S$ satisfies (*) iff for some finite subset $\left\{a_{1}, \ldots, a_{n}\right\} \subseteq S$, the ideal in $\operatorname{LUC}(S)$ generated by $\left\{\sum_{i=1}^{n} l_{a_{i}}\left(f-{ }_{s} f\right)\right.$; $s \in S, f \in \operatorname{LUC}(S)\}$ is not uniformly dense in $\operatorname{LUC}(S)$.

Received by the editors April 7, 1969.

(1) This research was supported in part by a postgraduate fellowship of the National Research Council of Canada. It contains parts of the results from the author's doctoral thesis at the University of British Columbia written under the direction of Professor E. E. Granirer. The author is most indebted to Professor Granirer for his valuable suggestions and encouragement during the preparation of the thesis. 
Combinatorial properties for topological semigroups satisfying $\left(^{*}\right)$ are also obtained (\$6). We show in $\S 3$ that there exist many topological semigroups $S$ for which LUC $(S)$ has a LIM in Co $\Delta(S)$ and yet $m(S)$ does not have a LIM.

When $S$ is discrete, $m(S)=\mathrm{LUC}(S)$ and $\Delta(S)=\beta S$, the Stone-Čech compactification of $S$. The idea in considering discrete semigroups satisfying $\left(^{*}\right)$ is due to J. Sorenson, who has shown in [19, Theorem 3.3.6] among other things the following interesting result: $m(S)$ has a LIM in Co $\beta S$ iff $S /(r)$ is a finite group $(S /(r)$ is the factor semigroup defined by the equivalence relation $(r): a(r) b$ iff $a c=b c$ for some $c \in S$ ). In $\S 5$, we show how some of the results of Granirer [4], [5] and Mitchell [14] on semigroups $S$ admitting a LIM in $\beta S$ can be generalised to semigroups $S$ admitting a LIM in Co $\beta S$. We show in particular that a discrete semigroup $S$ admits a LIM in Co $\beta S \Leftrightarrow(a)$ for some fixed $n$, and each finite subset $\sigma \subseteq S$, there exists $F_{\sigma} \subseteq S$, cardinality of $F_{\sigma}=n$, and $a F_{\sigma}=F_{\sigma}$ for all $a \in \sigma \Leftrightarrow(b)$ for some fixed $m$, there exists a net of finite means $\mu_{\alpha}=(1 / m) \sum_{1}^{m} p_{a_{i}^{\alpha}}$ such that $\left\|L_{a} \mu_{\alpha}-\mu_{\alpha}\right\| \rightarrow 0$ for all $a \in S$.

Finally, if $S$ is a topological semigroup, we raise in this paper the following problems:

(1) If $S$ satisfies $\left(^{*}\right)$, is every extreme point of the set of LIM on $\operatorname{LUC}(S)$ in Co $\Delta(S)$ ?

(2) If for some fixed $n$ and for each $f \in \operatorname{LUC}(S)$, the pointwise closure of $\left\{(1 / n) \sum_{i=1}^{n} r_{a_{i}}(f) ; a_{i} \in S\right\}$ has a constant function, does $S$ satisfy $\left(^{*}\right)$ ?

(3) If $S_{\alpha}, \alpha \in I$ are topological semigroups satisfying $(*)$ and $\operatorname{LUC}\left(S_{\alpha}\right)$ has a multiplicative LIM for all but a finite number of $\alpha$ in $I$, does the full direct product $\pi_{\alpha \in I} S_{\alpha}$ with the product topology satisfy $\left(^{*}\right)$ ? (Day, [1, p. 517].)

The answer to (1) is affirmative for $S$ discrete (Sorenson [19, Theorem 3.3.6]) and the answer to (2) is also affirmative for $n=1$ (Granirer and Lau [9, Theorem 2]). In this paper, we given an answer to (2) and (3) (Theorem 5.6 and Proposition 6.7) for discrete semigroups. But for the general case, all the three problems, to our best knowledge, are still open.

Several authors have recently studied topological semigroups $S$ for which the space LUC $(S)$ admits a LIM. Namioka in [17] studies topological semigroups $S$ for which $\operatorname{LUC}(S)$ has a $\mathrm{LIM}$ and Mitchell( $\left.{ }^{2}\right)$ [16] recently considers a certain fixed point property on topological semigroups $S$ for which $\operatorname{LUC}(S)$ has a multiplicative LIM.

2. Some notations. For any set $A$, we shall denote by $|A|$, the cardinality of $A$.

If $A$ is a subset of a semigroup $S, a \in S$, then $a^{-1} A=\{s \in S$; as $\in S\}$, and $1_{A}$ $\in m(S)$ such that

$$
\begin{aligned}
1_{A}(s)=1 & \text { if } s \in A, \\
=0 & \text { if } s \notin A .
\end{aligned}
$$

$\left({ }^{2}\right)$ The author is grateful to Professor T. Mitchell in providing him with a preprint of his paper [16]. 
Let $S$ and $T$ be topological semigroups, then $S \times T$ will denote the product topological semigroup with coordinatewise multiplication and product topology.

Let $S$ be a semigroup, $X$ a translation invariant normed closed subalgebra of $m(S)$ containing constants. Then $\varphi \in X^{*}$ is a point measure [finite mean] if $\varphi$ is the restriction of some point measure [finite mean] on $m(S)$. It is well known that the set of finite means [point measure] on $X$ is $w^{*}$-dense (i.e. $\left.\sigma\left(X^{*}, X\right)\right)$ in the set of means [multiplicative means] on $X$.

$X$ is left amenable (LA) if $X$ has a LIM and $X$ is extremely left amenable (ELA) if $X$ has a multiplicative LIM. $S$ is LA [ELA] iff $m(S)$ is LA [ELA]. (See Day [1], Granirer [4].)

Let $S$ be a topological semigroup, then $\operatorname{LUC}(S)$ is a translation invariant normed closed subalgebra of $m(S)$ containing constants. Furthermore, if $\varphi \in$ $\operatorname{LUC}(S)^{*}, f \in \operatorname{LUC}(S)$, then the function $h(s)=\varphi\left({ }_{s} f\right)$ is in $\operatorname{LUC}(S)$ (Namioka [17, p. 68]). Hence if $\varphi, \mu \in \operatorname{LUC}(S)^{*}$, we may define $\varphi \odot \mu \in \operatorname{LUC}(S)^{*}$ the Arens product of $\varphi$ and $\mu$ by $(\varphi \odot \mu)(f)=\varphi(h)$, where $h(s)=\mu\left({ }_{s} h\right)$ for $f \in \operatorname{LUC}(S)$ (Day $[1$, p. 540]). The Arens product renders the set of means on $\operatorname{LUC}(S)$ and $\Delta(S)$ into a semigroup. Furthermore, if $w^{*}-\lim \varphi_{\alpha}=\varphi_{0}, \varphi_{\alpha}, \varphi_{0} \in \operatorname{LUC}(S)^{*}$, then

(1) $w^{*}-\lim \left(\varphi_{\alpha} \odot \mu\right)=\varphi_{0} \odot \mu$ for all $\mu \in \operatorname{LUC}(S)^{*}$,

(2) $w^{*}-\lim \left(L_{a} \varphi_{\alpha}\right)=L_{a} \varphi_{0}$ for all $a \in S$.

For other properties of Arens product, we refer our reader to Day [1].

3. $N$-extreme amenability. For any topological semigroup $S, \operatorname{LUC}(S)$ is n-extremely left amenable ( $n$-ELA) if there exists a subset $H_{0}$ of $\Delta(S),\left|H_{0}\right|=n$, which is minimal with respect to the property: $L_{a} H_{0}=H_{0}$ for all $a \in S$.

REMARKS 3.1. (a) It is important to note that if $H_{1}$ is another finite subset of $\Delta(S)$ which is minimal with respect to the property: $L_{a} H_{1}=H_{1}$ for all $a \in S$, then $\left|H_{0}\right|=\left|H_{1}\right|$. In fact if $\varphi_{0} \in H_{0}$ is fixed, then by continuity of Arens product in the first variable (Day [1, p. 529]) $H_{1} \odot \varphi_{0} \subseteq H_{0}$. Let $F_{0}=\left\{L_{a} \psi_{0} ; a \in S\right\}$, where $\psi_{0} \in$ $H_{1} \odot \varphi_{0}$. Then $F_{0} \subseteq H_{1} \odot \varphi_{0} \subseteq H_{0}$, and $L_{a} F_{0}=F_{0}$ for all $a \in S$ since the restriction of $\left\{L_{a} ; a \in S\right\}$ to $H_{0}$ is a finite group. Hence $F_{0}=H_{1} \odot \varphi_{0}=H_{0}$ by minimality of $H_{0}$, and $\left|H_{1}\right| \geqq\left|H_{0}\right|$. Interchanging $H_{1}$ and $H_{0}$ in the above argument, we obtain $\left|H_{1}\right| \leqq\left|H_{0}\right|$.

(b) If $H_{0}=\left\{\varphi_{1}, \ldots, \varphi_{n}\right\}$, it is easy to see that $(1 / n) \sum_{1}^{n} \varphi_{i} \in \mathrm{Co} \Delta(S)$ is a LIM on $\operatorname{LUC}(S)$. Conversely if $\operatorname{LUC}(S)$ has a LIM in Co $\Delta(S)$, then $\operatorname{LUC}(S)$ is $m$-ELA for some $m$, since as known, finite subsets of $\Delta(S)$ are linearly independent; hence if $\mu=\sum_{1}^{n} \lambda_{i} \varphi_{i}, \varphi_{i}$ distinct elements in $\Delta(S)$, is a $\operatorname{LIM}$ on $\operatorname{LUC}(S)$, then $L_{a} F=F$ for all $a \in S, F=\left\{\varphi_{1}, \ldots, \varphi_{n}\right\}$. Then $F_{0}=\left\{L_{a} \varphi_{1} ; a \in S\right\} \subseteq F$ is minimal with respect to the property $L_{a} F_{0}=F_{0}$ for all $a \in S$ since the restriction of $\left\{L_{a} ; a \in S\right\}$ to $F$ is a finite group.

(c) It has been shown by Granirer and Lau in [9, Theorem 3] that if $G$ is a locally compact topological group, $S$ a subsemigroup of $G, \operatorname{LUC}(S)$ has a LIM in Co $\Delta(S)$ iff $S$ is a finite subgroup of $G$. Consequently the only subsemigroups $S$ of $G$ for which $\operatorname{LUC}(S)$ is $n$-ELA are the finite subgroups of $G$ of order $n$. 
A semigroup $S$ is $n$-extremely left amenable ( $n$-ELA) if $S$ is a discrete semigroup, $\operatorname{LUC}(S)=m(S)$ is $n$-ELA. For $n=1$, this coincides with the class of ELA semigroups which has been studied by Mitchell [14], and Granirer [4], [5] and [6].

Some examples of $n$-ELA semigroups are:

(1) Any finite group of order $n$ is $n$-ELA.

(2) Any left amenable finite semigroup is $n$-ELA for some $n$.

(3) If $S$ is any ELA semigroup, and $G$ a group of order $n$, then $S \times G$ is $n$-ELA (Proposition 6.4).

(4) Let $(T, \circ)$ be a semigroup, and $(G, *)$ a group of order $n$ such that $G \cap T$ $=\varnothing$. Let $S=G \cup T$ and define on $S$ the following binary operations

$$
\begin{aligned}
t_{1} \cdot t_{2} & =t_{1} \circ t_{2} & & \text { if } t_{1}, t_{2} \in T, \\
g_{1} \cdot g_{2} & =g_{1} * g_{2} & & \text { if } g_{1}, g_{2} \in G, \\
t \cdot g & =g \cdot t=g & & \text { for all } t \in T \text { and } g \in G .
\end{aligned}
$$

Then $S$ is $n$-ELA.

REMARK. Consider the special case of (4) when $T$ has just one element, and $G$ is a group of order $n>2$. Then $S$ is an $n$-ELA semigroup which is not isomorphic to a product semigroup $S_{0} \times G_{0}$, where $S_{0}$ is an ELA semigroup and $G_{0}$ is a group of order $n$, for otherwise $n+1=|S|=\left|S_{0} \times G_{0}\right|=k n$ for some $k$, which is impossible when $n>2$.

It is clear that if $S$ is an $n$-ELA semigroup, then for any topology defined on $S$ which is compatible with the structure, $\operatorname{LUC}(S)$ is $m$-ELA for some $m \leqq n$ (and $m$ divides $n$, Proposition 6.1). The following example shows that for any $n$, there exists a huge class of topological semigroup $S$, such that $\operatorname{LUC}(S)$ is $n$-ELA, but $S$ is not even left amenable as a discrete semigroup.

MAIN EXAMPLE. E. Hewitt has constructed a hausdorff regular topological space $S_{0}$ such that the only continuous real functions on $S_{0}$ are the constant functions [11]. Define on $S_{0}$ the binary operation $a \cdot b=a$ for $a, b \in S$. As shown by Granirer [8, p. 108], $S_{0}$ is a topological semigroup. Furthermore, $\operatorname{LUC}\left(S_{0}\right)$ $=C\left(S_{0}\right)$ is ELA (Mitchell [15, p. 123]). Let $T_{0}$ be any $n$-ELA (discrete) semigroup and $\tilde{T}_{0}=S_{0} \times T_{0}$. Then LUC $\left(\widetilde{T}_{0}\right)$ is $n$-ELA (Proposition 6.4) even though $\tilde{T}_{0}$ is not even left amenable as a discrete semigroup, since if $a, b \in \tilde{T}_{0}, a=\left(s_{1}, t_{1}\right)$, $b=\left(s_{2}, t_{2}\right)$ such that $s_{1} \neq s_{2}$, then $a \tilde{T}_{0} \cap b \tilde{T}_{0}=\varnothing$.

4. Structure theorems. In this section we shall prove our main results which give the basic structure of a topological semigroup $S$ for which $\operatorname{LUC}(S)$ is $n$-ELA.

An equivalence relation $E$ on a semigroup $S$ is two-sided stable if $a E b$ implies $c a E c b$ and $a c E b c$ for all $c \in S$ (Ljapin [12, p. 39]).

Let $S$ be a topological semigroup and $H \subseteq \Delta(S)$ such that $L_{a} H=H$ for all $a \in S$. Define on $S$ the two-sided stable equivalence relation $E$ : $a E b$ iff $L_{a} \varphi=L_{b} \varphi$ for all $\varphi \in H$ (Sorenson [19, Chapter 2]) and denote by $S / H$ the factor semigroup defined by this equivalence relation. 
THEOREM 4.1 (DECOMPOSITION). If $S$ is a topological semigroup such that $L U C(S)$ is $n$-ELA, then there exists a collection $\mathscr{F}$ of $n$ disjoint open and closed subsets of $S$, with union $S$, such that $1_{A} \in L U C(S)$ for all $A \in \mathscr{F}$ and $\mathscr{F}$ is the decomposition of $S$ by cosets of $S / H$ for any finite subset $H \subseteq \Delta(S)$ satisfying $L_{a} H=H$ for all $a \in S$.

Proof. Let $H_{0} \subseteq \Delta(S)$ such that $\left|H_{0}\right|=n$ and $L_{a} H_{0}=H_{0}$ for all $a \in S$. Then $S / H_{0}$ is a finite group of order $m$. Let $T=\left\{s \in S ; L_{s} \varphi=\varphi\right.$ for all $\left.\varphi \in H_{0}\right\} . T$ is a closed subsemigroup of $S$ and if $\left\{a_{1}, \ldots, a_{m}\right\}$ is a coset representative of $S / H_{0}, \mathscr{F}=$ $\left\{a_{i}^{-1} T ; i=1, \ldots, m\right\}$, then $S$ is the union of the $m$ disjoint open and closed sets in $\mathscr{F}$. If $m<n$, let $\varphi_{0} \in H_{0}, F_{0}=\left\{L_{a} \varphi_{0}, \ldots, L_{a_{m}} \varphi_{0}\right\}$, then $L_{a} F_{0}=F_{0}$ for all $a \in S$ and $\left|F_{0}\right|<\left|H_{0}\right|$, which is impossible. Hence $m \geqq n$. We shall show that $1_{T} \in \operatorname{LUC}(S)$ and for each $i=1, \ldots, m$, there exists $\varphi_{i} \in H_{0}$ such that $\varphi_{i}\left(1_{a_{i}^{-1} T}\right)=1$. Consequently $m=n$. Let $s_{\alpha} \rightarrow s_{0}, s_{\alpha}, s_{0} \in S$. If $s_{0} \in a_{k}^{-1} T$, then there exists $\alpha_{0}$ such that $s_{\alpha} \in a_{k}^{-1} T$ for all $\alpha \geqq \alpha_{0}$. Hence for all $\alpha \geqq \alpha_{0}, s_{\alpha}^{-1} T=s_{0}^{-1} T$ and $\left\|s_{\alpha}^{1} T-s_{0}^{1} T\right\|=0$ i.e. $1_{T} \in \operatorname{LUC}(S)$. Let $\varphi_{0} \in H_{0}$ be fixed, then $1=\varphi_{0}\left(1_{S}\right)=\sum_{1}^{n} \varphi_{0}\left(1_{a_{i}^{-1} T}\right)$ which implies $\varphi_{0}\left(1_{a_{i}^{-1} T}\right)>0$ for some $k$. Since $\varphi_{0}$ is multiplicative, $\varphi_{0}\left(1_{a_{k}^{-1} T}\right)=1$. Hence for each $i=1, \ldots, m$, if $\varphi_{i}=L_{b_{i} a_{k}} \varphi_{0}$, where $a_{i} b_{i} \in T$, then $\varphi_{i}\left(1_{a_{i}{ }^{-1} T}\right)=1$.

To complete the proof of the theorem, it suffices to show that for any two finite subsets $H_{1}$ and $H_{2}$ of $\Delta(S)$ such that $L_{s} H_{i}=H_{i}$ for all $s \in S, i=1,2$, if $a, b \in S$, then $L_{a} \varphi=L_{b} \varphi$ for all $\varphi \in H_{1}$ iff $L_{a} \psi=L_{b} \psi$ for all $\psi \in H_{2}$. Assume that $L_{a} \varphi=L_{b} \varphi$ for all $\varphi \in H_{1}$ and let $\varphi_{0} \in H_{1}$ be fixed, $p_{s_{\alpha}}$ be a net of point measures on $\operatorname{LUC}(S)$ such that $w^{*}-\lim p_{s_{\alpha}}=\varphi_{0}$. If $\psi_{0} \in H_{2}$, for each $\alpha$, there exists $\psi_{\alpha} \in H_{2}$ such that $p_{s_{\alpha}} \odot \psi_{\alpha}=L_{s_{\alpha}} \psi_{\alpha}=\psi_{0}$. By finiteness of $H_{0}$, we may assume (by taking subnet if necessary) that there exists $\alpha_{0}$ and $\gamma_{0} \in H_{2}$ such that $\psi_{\alpha}=\gamma_{0}$ for all $\alpha \geqq \alpha_{0}$. Hence $p_{s_{\alpha}} \odot \gamma_{0}=\psi_{0}$ for all $\alpha \geqq \alpha_{0}$ and $\varphi_{0} \odot \gamma_{0}=\psi_{0}$ (Day [1, p. 529]). Consequently, $L_{a} \psi_{0}$ $=L_{a}\left(\varphi_{0} \odot \psi_{0}\right)=\left(L_{a} \varphi_{0}\right) \odot \psi_{0}=\left(L_{b} \varphi_{0}\right) \odot \psi_{0}=L_{b} \psi_{0}$. The converse follows from interchanging $\mathrm{H}_{1}$ and $\mathrm{H}_{2}$.

Corollary. For any connected topological semigroup S, if LUC(S) has a LIM in $\operatorname{Co} \Delta(S)$, then $L U C(S)$ is ELA.

REMARK 4.2. Let $S$ be a topological semigroup such that $\operatorname{LUC}(S)$ is $n$-ELA, $\mathscr{F}$ the decomposition of $S$ as obtained in Theorem 4.1. We have shown in the proof of Theorem 4.1 that there exists an open and closed subsemigroup $T \in \mathscr{F}$ such that $1_{T} \in \mathrm{LUC}(S)$ and if $H$ is any finite subset of $\Delta(S), L_{a} H=H$ for all $a \in S$, then $T=\left\{s \in S ; L_{s} \varphi=\varphi\right.$ for all $\left.\varphi \in H\right\}$. Furthermore, if $\left\{a_{1}, \ldots, a_{n}\right\}$ is a coset representative of $S / H_{0}$, then $\mathscr{F}=\left\{a_{i}^{-1} T ; i=1, \ldots, n\right\}$. Consequently, $\varphi_{0}\left(1_{T}\right)=1$ for some $\varphi_{0} \in H$ and $\mu\left(1_{T}\right)=1 / n$ for all $\operatorname{LIM} \mu$ on $\operatorname{LUC}(S)$.

Let $\operatorname{PLUC}(S)=\{P f ; f \in \operatorname{LUC}(S)\}$, where $P f(s)=f(s)$ if $s \in T$. Then $\operatorname{PLUC}(S)$ is a norm closed translation invariant subalgebra of $\mathrm{LUC}(T)$ containing $1_{T}$ as readily checked. Furthermore, if $S$ satisfies one of the following conditions, then $\operatorname{PLUC}(S)$ 
$=\operatorname{LUC}(T)$, (however we do not know whether or not $\operatorname{PLUC}(S)=\operatorname{LUC}(T)$ in general $)\left({ }^{3}\right)$ :

(a) $S$ is discrete.

(b) $\mathscr{F}=\left\{a_{i} T ; i=1, \ldots, n\right\}$ for some finite subset $\sigma=\left\{a_{1}, \ldots, a_{n}\right\} \subseteq S$ (this would be the case, for example, if $S$ had no proper right ideal).

Condition (a) is trivial. To show (b), we first note that $\left\{a_{1}, \ldots, a_{n}\right\}$ is necessarily a coset representative of $S / H$. Let $f \in \operatorname{LUC}(S)$, if we can show that $\pi f \in \operatorname{LUC}(S)$, where $(\pi f)(s)=f(s)$ if $s \in T$ and 0 otherwise, then $f=P(\pi f)$, and $\operatorname{PLUC}(S)=\operatorname{LUC}(T)$. Let $\left\{s_{\alpha}\right\}$ be a net in $S, s_{\alpha} \rightarrow s_{0}, s_{0} \in S$. Choose $a_{k} \in \sigma$ such that $s_{0} a_{k} \in T$. Then $s_{\alpha} a_{k} \rightarrow s_{0} a_{k}$. Since $T$ is open, there exists $\alpha_{0}$ such that $s_{\alpha} a_{k} \in T$ for all $\alpha \geqq \alpha_{0}$. Hence

$$
\begin{aligned}
\sup _{t \in S}\left|s_{s_{\alpha}}(\pi f)(t)-{ }_{s_{0}}(\pi f)(t)\right| & =\sup _{t \in S_{k}}\left|f\left(s_{\alpha} t\right)-f\left(s_{0} t\right)\right| \\
& =\sup _{t \in T}\left|f\left(s_{\alpha} a_{k} t\right)-f\left(s_{0} a_{k} t\right)\right| \rightarrow 0 .
\end{aligned}
$$

Consequently, $\pi f \in \operatorname{LUC}(S)$.

Lemma 4.3. PLUC(S) has a multiplicative LIM.

Proof. By Remark 4.2, there exists $\varphi_{0} \in \Delta(S)$ such that $L_{t} \varphi_{0}=\varphi_{0}$ for all $t \in T$ and $\varphi_{0}\left(1_{T}\right)=1$. For $f \in \operatorname{LUC}(S)$, define $\mu_{0}(P f)=\varphi_{0}\left(1_{T} f\right)$. Then $\mu_{0}$ is a multiplicative mean on $\operatorname{PLUC}(S)$, and if $t \in T$, then $\mu_{0}\left({ }_{t}(P f)\right)=\mu_{0}\left(P\left({ }_{t} f\right)\right)=\varphi_{0}\left(1_{T}\left({ }_{t} f\right)\right)=\varphi_{0}\left({ }_{t}\left(1_{T} f\right)\right)$ $=\varphi_{0}\left(1_{T} f\right)=\mu_{0}(P f)$ since $t^{-1} T=T$.

Notation. For any topological semigroup $S$, if $\operatorname{LUC}(S)$ is $n$-ELA, then $\operatorname{PLUC}(S) \subseteq \operatorname{LUC}(T)$ will denote the algebra of functions as defined in Remark 4.2.

THEOREM 4.4. For any topological semigroup $S$, if $L U C(S)$ is $n-E L A$, then there exists a linear transformation mapping the set of LIM on PLUC $(S)$ one-one onto the set of LIM on LUC $(S)$.

Proof. Let $H$ be a finite subset of $\Delta(S)$ such that $L_{a} H=H$ for all $a \in S, T=$ $\left\{s \in S, L_{s} \varphi=\varphi\right.$ for all $\left.\varphi \in H\right\}$ and $P: \operatorname{LUC}(S) \rightarrow \operatorname{LUC}(T)$ where $(P f)(s)=f(s)$ if $s \in T$. For any coset representative $\sigma$ of $S / H$, define the mapping $F_{\sigma}:[\operatorname{PLUC}(S)]^{*}$ $\rightarrow \operatorname{LUC}(S)^{*}$ by $F_{\sigma}(\varphi)=(1 / n) \sum_{a \in \sigma} L_{a} P^{*}(\varphi)$. We shall show that $F_{\sigma_{0}}$ is the required linear transformation for $\sigma_{0}$, a fixed coset representative of $S / H$.

Choose $\varphi_{0} \in H$ such that $\varphi_{0}\left(1_{T}\right)=1$ (Remark 4.2) and $\left\{p_{t_{\alpha}}\right\}$ be a net of point measures on $\operatorname{LUC}(S)$ such that $w^{*}-\lim p_{t_{\alpha}}=\varphi_{0}$. If $\psi$ is a $\operatorname{LIM}$ on $\operatorname{PLUC}(S)$

( $\left.{ }^{3}\right)$ The following example from Mitchell [16] shows that if $T$ is a subsemigroup of a topological semigroup $S$, then the algebra $A=\{P f ; f \in \operatorname{LUC}(S)\} \subseteq \operatorname{LUC}(S)$ where $P f(s)=f(s)$ if $s \in S$, need not coincide with $\operatorname{LUC}(T)$ : let $(T,+)$ be the semigroup of positive integers with addition and $S=T \cup\{a\}$, where $a \notin T$, be the one point compactification of $T$. Define on $S$ the binary operation $s_{1} \cdot s_{2}=s_{1}+s_{2}$ if $s_{1}, s_{2} \in T$ and $s a=a s=s$ for all $s \in S$. Then $S$ is a compact topological semigroup. The function $g(n)=\sin (n)$ is in $\operatorname{LUC}(T)=m(T)$, but $P f \neq g$ for any $f \in \operatorname{LUC}(S)$. 
(Lemma 4.3), then $p_{t} \odot P^{*} \psi=P^{*} \psi$ for all $t \in T$. Hence

$$
\varphi_{0} \odot P^{*} \psi=w^{*}-\lim _{\alpha}\left(p_{t_{\alpha}} \odot P^{*} \psi\right)=P^{*} \psi
$$

(Day [1, p. 529]). If $a, b \in S$ such that $L_{a} \varphi=L_{b} \varphi$ for all $\varphi \in H$, then

$$
\begin{aligned}
L_{a} P^{*} \psi & =L_{a}\left(\varphi_{0} \odot P^{*} \psi\right)=\left(L_{a} \varphi_{0}\right) \odot P^{*} \psi=\left(L_{b} \varphi_{0}\right) \odot P^{*} \psi \\
& =L_{b}\left(\varphi_{0} \odot P^{*} \psi\right)=L_{b} P^{*} \psi .
\end{aligned}
$$

Hence for any coset representative $\sigma_{1}, \sigma_{2}$ of $S / H, F_{\sigma_{1}}(\psi)=F_{\sigma_{2}}(\psi)$. Consequently, if $f \in \operatorname{LUC}(S), s \in S$, then $F_{\sigma_{0}}(\psi)\left({ }_{s} f\right)=F_{s \sigma_{0}}(\psi)(f)=F_{\sigma_{0}}(\psi)(f)$ i.e. $F_{\sigma_{0}} \psi$ is a LIM on $\operatorname{LUC}(S)$.

Conversely if $\mu$ is a $\operatorname{LIM}$ on $\operatorname{LUC}(S)$, then $\mu\left(1_{T}\right)=1 / n$ (Remark 4.2). Define $\psi$ on $\operatorname{PLUC}(S)$ by $\psi(g)=n \cdot \mu\left(1_{T} f\right)$ if $g=P f$. If $t \in T, f \in \operatorname{LUC}(S)$, then $\psi(t(P f))$ $=\psi\left(P\left({ }_{t} f\right)\right)=n \cdot \mu\left(1_{T}(t f)\right)=n \cdot \mu\left({ }_{t}\left(1_{T} f\right)\right)=n \cdot \mu\left(1_{T} f\right)=\psi(P f)$ since $t^{-1} T=T$. Hence $\psi$ is a LIM on $\operatorname{PLUC}(S)$. Furthermore, $F_{\sigma_{0}}(\psi)=\mu$ since if $f \in \operatorname{LUC}(S), \sigma_{0}=\left\{a_{1}, \ldots, a_{n}\right\}$ and $b_{i} \in S$ such that $b_{i} a_{i} \in T$, then

$$
\begin{aligned}
F_{\sigma_{0}}(\psi)(f) & =\frac{1}{n} \sum_{i=1}^{n} n \cdot \mu\left(1_{T}\left(a_{i} f\right)\right)=\frac{1}{n} \sum_{i=1}^{n} n \cdot \mu\left(a_{i}\left(1_{b_{i}^{-1} T} f\right)\right) \\
& =\frac{1}{n} \sum_{i=1}^{n} n \cdot \mu\left(1_{b_{i}}{ }^{-1} T\right)=\mu(f) .
\end{aligned}
$$

To see that $F_{\sigma_{0}}$ is one-one, we first observe that if $\psi$ is a LIM on $\operatorname{PLUC}(S)$, $f \in \operatorname{LUC}(S)$, then $F_{\sigma_{0}}(\psi)\left(1_{T} f\right)=(1 / n) \psi\left(P\left(1_{T} f\right)\right)$. Hence if $\psi_{1}$ and $\psi_{2}$ are LIM on $\operatorname{PLUC}(S), f \in \operatorname{LUC}(S)$ such that $\psi_{1}(P f) \neq \psi_{2}(P f)$, then $\psi_{1}\left(P\left(1_{T} f\right)\right) \neq \psi_{2}\left(P\left(1_{T} f\right)\right)$ and hence $F_{\sigma_{0}}\left(\psi_{1}\right) \neq F_{\sigma_{0}}\left(\psi_{2}\right)$.

REMARK 4.5. Let $S$ be a topological semigroup such that $\operatorname{LUC}(S)$ is $n$-ELA, $H$ a finite subset of $\Delta(S)$ such that $L_{a} H=H$ for all $a \in S, T=\left\{s \in S ; L_{s} \varphi=\varphi\right.$ for all $\varphi \in H\}$. We have shown in Theorem 4.4 that if $\varphi_{0}$ is a multiplicative LIM on $\operatorname{PLUC}(S)$ (Lemma 4.3), then $(1 / n) \sum_{a \in \sigma_{0}} L_{a}\left(P^{*} \varphi_{0}\right) \in \operatorname{Co} \Delta(S)$ is a $\operatorname{LIM}$ on LUC(S) for any coset representative $\sigma_{0}$ of $S / H$, and $(P f)(t)=f(t)$ for $t \in T$.

It has been proved by Granirer [5, Theorem 6] that if $S$ is ELA then every extreme point of the set of LIM on $m(S)$ is multiplicative. Theorem 4.4 yields the following generalization of Granirer's result which is due to Sorenson [19, Theorem 3.3.6]:

COROLlaRY (SORENSON). If $S$ is an $n$-ELA (discrete) semigroup, then every extreme point of the set of LIM on $m(S)$ is of the form $(1 / n) \sum_{1}^{n} \gamma_{i}, \gamma_{i} \in \beta S$.

Proof. $\operatorname{PLUC}(S)=m(T)$ for some ELA subsemigroup $T$ of $S$ (Remark 4.2, Lemma 4.3). Hence every extreme point of the set of LIM on $\operatorname{PLUC}(S)$ is multiplicative (Granirer [5, Theorem 6]). The assertion now follows from Theorem 4.4 and Remark 4.5.

REMARK 4.6. In view of Sorenson's result, it is natural for us to ask the following question: if $S$ is a topological semigroup such that $\operatorname{LUC}(S)$ is $n$-ELA, then does 
every extreme point of the set of $\operatorname{LIM}$ on $\operatorname{LUC}(S)$ have the form $(1 / n) \sum_{1}^{n} \gamma_{i}$, where $\gamma_{i} \in \Delta(S)$ ? We do not know the answer even for $n=1$.

LEMMA 4.7. For any topological semigroup $S$, if LUC(S) has a LIM of the form $(1 / n) \sum_{1}^{n} \varphi_{i}$, where $\varphi_{i} \in \Delta(S)$ (not necessarily distinct), then $L U C(S)$ is $m$-ELA for some $m \leqq n, m$ divides $n$.

Proof. LUC $(S)$ is $m$-ELA for some $m$ (Remark 3.1). If $H$ is a finite subset of $\Delta(S)$ such that $L_{a} H=H$ for all $a \in S$, then $S / H$ is a group of order $m$ (Theorem 4.1). Let $\pi$ be the natural homomorphism from $S$ onto $S / H$, then $\pi\left(s_{\alpha}\right) \rightarrow \pi\left(s_{0}\right)$ whenever $s_{\alpha} \rightarrow s_{0}, s_{\alpha}, s_{0} \in S$. Hence $f \circ \pi \in \operatorname{LUC}(S)$ for $f \in m(S / H)$. Define $\tilde{\phi}_{i}(f)$ $=\varphi_{i}(f \circ \pi)$ for all $f \in m(S / H)$. Then $(1 / n) \sum_{1}^{n} \tilde{\phi}_{i}$ is a LIM on $S / H_{0}$. By uniqueness of LIM on a finite group, we have $m \leqq n$, and $m$ divides $n$.

Let $S$ be a topological semigroup for any finite subset $\sigma_{0} \subseteq S$, denote by

$$
J\left(\sigma_{0}\right)=\text { ideal in } \operatorname{LUC}(S) \text { generated by }\left\{\sum_{a \in \sigma_{0}} l_{a}\left(f-{ }_{s} f\right) ; f \in \operatorname{LUC}(S), s \in S\right\} \text {. }
$$

THEOREM 4.8. For any topological semigroup $S$, if $L U C(S)$ is $n$-ELA, then for some $\sigma_{0} \subseteq S,\left|\sigma_{0}\right|=n, J\left(\sigma_{0}\right)$ is not uniformly dense in LUC $(S)$. Conversely, if there exists $\sigma_{0} \subseteq S,\left|\sigma_{0}\right|=n$ such that $J\left(\sigma_{0}\right)$ is not uniformly dense in $L U C(S)$, then $L U C(S)$ is $m$-ELA for some $m \leqq n, m$ divides $n$.

Proof. If $\operatorname{LUC}(S)$ is $n$-ELA, by Remark 4.5, there is some $\sigma_{0} \subseteq S,\left|\sigma_{0}\right|=n$ and $\gamma_{0} \in \Delta(S)$ such that $\mu=(1 / n) \sum_{a \in \sigma_{0}} L_{a} \gamma_{0}$ is a LIM on LUC $(S)$. Hence if $h=$ $g\left(\sum_{a \in \sigma_{0}} l_{a}\left(f-{ }_{s} f\right)\right), f, g \in \operatorname{LUC}(S), s \in S$, then $\mu(h)=0$. Since $\mu\left(1_{S}\right)=1, J\left(\sigma_{0}\right)$ is not uniformly dense in $\operatorname{LUC}(S)$. Conversely, if $J\left(\sigma_{0}\right)$ is not uniformly dense in $\operatorname{LUC}(S)$, then there exists $\varphi_{0} \in \Delta(S)$ such that $\varphi_{0}\left(J\left(\sigma_{0}\right)\right)=0$ (see proof of Lemma 3 [5, p. 99]). Hence, $(1 / n) \sum_{a \in \sigma_{0}} L_{a} \varphi_{0}$ is a $\operatorname{LIM}$ on $\operatorname{LUC}(S)$. Now apply Lemma 4.7.

REMARK. It follows from Theorem 2 of Granirer [5] that $\operatorname{LUC}(S)$ has a multiplicative LIM iff the ideal in $\operatorname{LUC}(S)$ generated by $\left\{f-{ }_{s} f ; f \in \operatorname{LUC}(S), s \in S\right\}$ is not uniformly dense in $\mathrm{LUC}(S)$. Theorem 4.8 partially generalises this result.

5. $N$-extremely amenable semigroups. In this section we begin our investigation for the class of $n$-ELA semigroups and obtain results which generalise those of Mitchell [14] and Granirer [4], [5] for the class of ELA semigroups.

Let $S$ be a semigroup with finite intersection property for right ideals (f.i.p.r.i.). Denote by $S /(r)$ the factor semigroup defined by the two-sided stable equivalence relation $(r)$ : for any $a, b \in S, a(r) b$ iff $a c=b c$ for some $c \in S$. As known, $S /(r)$ is right cancellative (see for example [3, p. 372]).

REMARK 5.1. Let $S$ be a semigroup with f.i.p.r.i., such that $S /(r)$ is a group, and $S_{0}=\{s \in S$, homomorphic image of $s$ in $S /(r)$ is the identity $\}$. Then, as readily checked, for any $a, b \in S, a(r) b$ iff $a t=b t$ for some $t \in S_{0}$. Consequently $S_{0}$ is an ELA subsemigroup of $S$ (Mitchell [14, Corollary 3). Furthermore, if $S /(r)$ is finite and $\sigma_{1}, \sigma_{2}$ are coset representatives of $S /(r)$, then $\sigma_{1} t=\sigma_{2} t$ for some $t \in S_{0}$. 
The following result, which follows from the proof of Theorem 3.3.6 in Sorenson [19], shows an important relation between an $n$-ELA semigroup $S$ and the factor semigroup $S /(r)$. For the sake of completeness, we give a proof.

TheOREM 5.2 (SORENSON). For any semigroup $S, S$ is $n$-ELA iff $S$ has f.i.p.r.i. and $S /(r)$ is a group of order $n$.

Proof. If $S$ is $n$-ELA, let $H$ be a finite subset of $\beta S$ such that $L_{a} H=H$ for all $a \in S$. We shall show that for any $a, b \in S, L_{a} \varphi=L_{b} \varphi$ for all $\varphi \in H$ iff $a(r) b$; consequently $S /(r)$ is a group of order $n$ by Theorem 4.1. If $L_{a} \varphi=L_{b} \varphi$ for all $\varphi \in H$, let $s_{0} \in S$ such that $a s_{0}, b s_{0} \in T$, where $T=\left\{s \in S ; L_{s} \varphi=\varphi\right.$ for all $\left.\varphi \in H\right\}$ is an ELA subsemigroup of $S$ (Remark 4.2 and Lemma 4.3). Hence $a s_{0} t_{0}=b s_{0} t_{0}$ for some $t_{0} \in T$ (Granirer [4, Theorem 1]) i.e. $a(r) b$. If $a c=b c$ for some $c \in S$, then $L_{a}\left(L_{c} \varphi\right)$ $=L_{b}\left(L_{c} \varphi\right)$ for all $\varphi \in H$, which implies $L_{a} \varphi=L_{b} \varphi$ for all $\varphi \in H\left(=L_{c} H\right)$. Conversely, if $S /(r)$ is a group of $n$ elements, and $\varphi_{0}$ a multiplicative LIM on $m\left(S_{0}\right)$, where $S_{0}=\{s \in S$; homomorphic image of $s$ in $S /(r)$ is the identity $\}$, define $\tilde{\varphi}_{0} \in \beta S$ by $\tilde{\varphi}_{0}(f)=\varphi_{0}(\tilde{f})$, where $\tilde{f}(s)=f(s)$ if $s \in S_{0}$. If $\sigma_{0}$ is a coset representative of $S /(r)$, then as readily checked, $(1 / n) \sum_{a \in \sigma_{0}} L_{a} \tilde{\varphi}_{0}$ is a LIM on $m(S)$. Apply Lemma 4.7.

We now generalise a well-known result of Granirer [4, Theorem 1]: A semigroup $S$ is ELA iff any two elements has a common right zero.

THEOREM 5.3. For any semigroup $S$ and fixed $n$ :

(a) If $S$ is $n-E L A$ and $F_{0}$ is a coset representative of $S /(r)$, then for each finite subset $\sigma$ of $S$, there exists $t_{\sigma} \in S$, depending on $\sigma$, such that $a F_{0} t_{\sigma}=F_{0} t_{\sigma}$ for all $a \in \sigma$.

(b) If for any finite subset $\sigma$ of $S$, there exists $F_{\sigma} \subseteq S,\left|F_{\sigma}\right|=n$, such that a $F_{\sigma}=F_{\sigma}$ for all $a \in S$, then $S$ is $m$-ELA for some $m \leqq n, m$ dividing $n$.

Proof. (a) For each $a \in \sigma$, let $t_{a} \in S_{0}$ such that $a F_{0} t_{a}=F_{0} t_{a}$, where $S_{0}=\{s \in S$; homomorphic image of $s$ in $S /(r)$ is the identity is an ELA subsemigroup of $S$ (Remark 5.1). If $t_{\sigma} \in S_{0}$ such that $t_{a} t_{\sigma}=t_{\sigma}$ for all $a \in \sigma$ [14, Theorem 1] then $a F_{0} t_{\sigma}=F_{0} t_{\sigma}$ for all $a \in \sigma$.

(b) Let $\mathscr{F}=\{\sigma: \sigma$ finite subset of $S\}$ be directed by upward inclusion. If for each $\sigma \in \mathscr{F}, F_{\sigma}=\left\{a_{1}^{\sigma} \cdots a_{n}^{\sigma}\right\}$, let $\varphi_{i}$ be a cluster point of $p_{a_{i}^{\sigma}}$. Then, as readily checked, $(1 / n) \sum_{1}^{n} \varphi_{i}$ is a LIM on $m(S)$. The result now follows from Lemma 4.7.

REMARK 5.4. In order for a semigroup $S$ to be $m$-ELA for some $m \leqq n, n$ fixed, it is sufficient that for each finite subset $\sigma \subseteq S$, there exists $F_{\sigma} \subseteq S,\left|F_{\sigma}\right| \leqq n$ and $a F_{\sigma}=F_{\sigma}$ for all $a \in \sigma$ : Clearly $S$ has f.i.p.r.i. If $\sigma$ is a finite subset of $S,|\sigma|>n$, then $\sigma s_{0} \subseteq F_{\sigma}$ for $s_{0} \in F_{\sigma}$. Hence $a s_{0}=b s_{0}$ for some $a, b \in \sigma$. Consequently $S /(r)$ has at most $n$ elements.

For the class of $n$-ELA semigroups, Day's strong amenability theorem [1, Theorem 1] assumes a much nicer form:

THEOREM 5.5. For any semigroup $S$ and fixed $n$ : 
(a) If $S$ is $n-E L A$ and $F_{0}$ is a coset representative of $S /(r)$, then there exists a net $\psi_{\alpha}=(1 / n) \sum_{s \in F_{0}} p_{s t_{\alpha}}$ such that $\left\|L_{a} \psi_{\alpha}-\psi_{\alpha}\right\| \rightarrow 0$ for all $a \in S$.

(b) If there exists a net $\psi_{\alpha}=(1 / n) \sum_{1}^{n} p_{a_{i}^{\alpha}}$ such that $\left\|L_{a} \psi_{\alpha}-\psi_{\alpha}\right\| \rightarrow 0$ for all $a \in S$, then $S$ is $m$-ELA for some $m \leqq n, m$ divides $n$.

Proof. (a) Let $\mu_{\alpha}$ be a net of finite means on $m(S)$ such that $\left\|L_{a} \mu_{\alpha}-\mu_{\alpha}\right\| \rightarrow 0$ for all $a \in S$ [1, Theorem 1]. For each $\alpha$, let $t_{\alpha} \in S$ be such that $a F_{0} t_{\alpha}=F_{0} t_{\alpha}$ for all $a \in\left\{s \in S ; \mu_{\alpha}\left(1_{s}\right)>0\right\}$ (Theorem 5.4), and $\psi_{\alpha}=(1 / n) \sum_{a \in F_{0}} p_{s t_{\alpha}}$. Then for each $\alpha$, $\mu_{\alpha} \odot \psi_{\alpha}=\psi_{\alpha}$ and hence

$$
\left\|L_{a} \psi_{\alpha}-\psi_{\alpha}\right\|=\left\|L_{a}\left(\mu_{\alpha} \odot \psi_{\alpha}\right)-\left(\mu_{\alpha} \odot \psi_{\alpha}\right)\right\| \leqq\left\|L_{a} \mu_{\alpha}-\mu_{\alpha}\right\|\left\|\psi_{\alpha}\right\| \rightarrow 0
$$

for all $a \in S$ (Day, [1, p. 528]).

(b) Let $\varphi_{i}$ be a cluster point of $p_{a_{l}^{\alpha}}$. Then $(1 / n) \sum_{1}^{n} \varphi_{i}$ is a LIM on $m(S)$. The result follows from Lemma 4.7.

Remark. (a) For $n=1$, Theorem 5.5 is due to Granirer [4, Theorem 2].

(b) The following example shows that " $m \leqq n$ and $m$ divides $n$ " in Theorem 5.3 and Theorem 5.5 cannot be replaced by " $m=n$ " in general: consider the ELA semigroup $S=\left\{e_{1}, \ldots, e_{n}\right\}$ where $e_{i} \cdot e_{j}=e_{j}, i, j=1 \cdots n, F=S$ and $\mu_{0}=(1 / n) \sum_{1}^{n} p_{e_{i}}$. Then $a F=F$ and $\left\|L_{a} \mu_{0}-\mu_{0}\right\|=0$ for all $a \in S$.

It has been proved by Mitchell [13, Theorem 1] that a semigroup $S$ has a LIM iff for each $f \in m(S)$, the pointwise closure of $\operatorname{Co}\left\{r_{a} f ; a \in S\right\}$ contains a constant function. The following theorem, which generalises in part Theorem 1 of Granirer [5], is an analogue of Mitchell's result:

THEOREM 5.6. For any semigroup $S$ and fixed $n$ :

(a) If $S$ is $n$-ELA, then for each coset representative $F_{0}$ of $S /(r), f \in m(S)$, the pointwise closure of $\left\{(1 / n) \sum_{a \in F_{0}} r_{a t}(f) ; t \in S\right\}$ has a constant function.

(b) If for each $f \in m(S)$, the pointwise closure of $\left\{(1 / n) \sum_{i=1}^{n} r_{a_{i}}(f), a_{i} \in S\right\}$ has a constant function, then $S /(r)$ is a left amenable torsion group such that the order of each element divides $n$.

Proof. (a) As shown in the proof of Theorem 5.2, there exists $\varphi_{0} \in \beta S$ such that $(1 / n) \sum_{a \in F_{0}} L_{a} \varphi_{0}$ is a LIM on $m(S)$. Let $\left\{p_{t_{\alpha}}\right\}$ be a net of point measures on $S$ such that $w^{*}-\lim _{\alpha} p_{t_{\alpha}}=\varphi_{0}$. Then for each $f \in m(S)$,

$$
\frac{1}{n} \sum_{a \in F_{0}} r_{a t_{\alpha}}(f)(t)=\frac{1}{n} \sum_{a \in F_{0}} L_{a} p_{t_{\alpha}}\left({ }_{t} f\right)
$$

which converges to $(1 / n) \sum_{a \in \sigma_{0}} L_{a} \varphi_{0}(f)$ for all $t \in S$.

(b) The condition implies that $S$ is left amenable (Mitchell [13, Theorem 1]) and hence $S /(r)$ is a right cancellative left amenable semigroup (Granirer [3, p. 372]). Let $\bar{A} \subseteq S /(r), A=\{s \in S ; \bar{s} \in \bar{A}\}$, where $\bar{s}$ is the homomorphic image of $s$ in $S /(r)$, and $c \cdot 1$ a constant function in the pointwise closure of $\left\{(1 / n) \sum_{1}^{n} r_{a_{1}}\left(1_{A}\right)\right.$; $\left.a_{i} \in S\right\}$. Then $c=k / n$ for some $k=0,1, \ldots, n$. Let $\mu$ be a LIM on $m(S)$ such that $\mu\left(1_{A}\right)=k / n[13$, Theorem 1] and $\bar{\mu}$ be a LIM on $S /(r)$ defined by $\mu(f)=\mu(f \circ \pi)$, 
where $f \in m(S /(r))$ and $\pi$ is the natural homomorphism of $S$ onto $S /(r)$. Then $\bar{\mu}\left(1_{\bar{A}}\right)=k / n$. Hence we have shown that for any subset $\bar{A} \subseteq S /(r)$, there exists a LIM $\bar{\mu}$ on $S /(r)$ such that $\bar{\mu}\left(1_{\bar{A}}\right)=k / n$ for some $k=0,1, \ldots, n$. Consequently, the order of each element in $S /(r)$ is finite, since if $S /(r)$ has an element of infinite order, then for each rational number $0 \leqq \lambda \leqq 1$, there exists a subset $\bar{A} \subseteq S /(r)$ such that $\bar{\mu}\left(1_{\bar{A}}\right)=\lambda$ for all LIM $\bar{\mu}$ on $S /(r)$. (Granirer [4, p. 182]) which is impossible. If $S /(r)$ has an element of order $p$, then there exist $\bar{A} \subseteq S /(r)$ such that $\bar{\mu}\left(1_{\bar{A}}\right)=1 / p$ for all LIM $\bar{\mu}$ on $S /(r)[4$, p. 182]. Consequently, $1 / p=k / n$ and $p$ divides $n$. By Lemma 4 Granirer [7], $S /(r)$ is necessarily a group.

ReMARK. Let $S$ be a topological semigroup such that $\operatorname{LUC}(S)$ is $n$-ELA, and $H$ a finite subset of $\Delta(S)$ such that $L_{a} H=H$ for all $a \in S$; it is easy to see that (using Remark 4.5) for each $F_{0}$ coset representative of $S / H$, and $f \in \operatorname{LUC}(S)$, the pointwise closure of $\left\{(1 / n) \sum_{a \in F_{0}} r_{a t}(f) ; t \in S\right\}$ has a constant function. In fact, using Lemma 3 of Granirer and Lau [9], even the $\tau_{c}$ closure of $\left\{(1 / n) \sum_{a \in F_{0}} r_{a t}(f) ; t \in S\right\}$ has a constant function $\left(\tau_{c}\right.$ is the topology of uniform convergence on compacta). Conversely, if for each $f \in \operatorname{LUC}(S)$, the pointwise closure of $\left\{(1 / n) \sum_{1}^{n} r_{a_{i}}(f) ; a_{i} \in S\right\}$ has a constant function, then, as known, $\operatorname{LUC}(S)$ has a LIM [9, Theorem 2]. But is $S m$-ELA for some $m \leqq n$ ? For $n=1$, the answer is affirmative as proved by Granirer and Lau in [9]. However for $n>1$, the problem still remains open even when $S$ is discrete.

The next series of results is concerned with the $n$-extreme amenability of certain subsemigroups of $n$-ELA semigroups. Our proofs are algebraic using heavily characterizations obtained in Theorem 5.2 and Theorem 5.4.

Mitchell shows that [13, Theorem 9] if $T$ is a left thick subsemigroup of a semigroup $S$ (i.e. for each finite subset $\sigma$ of $S, \sigma s_{\sigma} \subseteq T$ for some $\left.s_{\sigma} \in S\right\}$, then $T$ is LA iff $S$ is LA. The following is an analogue of this result:

Proposition 5.7. If $T$ is a left thick subsemigroup of a semigroup $S$, then $T$ is $n$-ELA iff $S$ is $n$-ELA.

Proof. If $T$ is $n$-ELA, $F_{0}$ a coset representative of $T /(r)$ and $\sigma$ a finite subset of $S$, let $t_{0} \in T$ such that $\sigma t_{0} \subseteq T\left[13\right.$, p. 256] and $t_{\sigma} \in T$ such that $b F_{0} t_{\sigma}=F_{0} t_{\sigma}$ for all $b \in \sigma t_{0}$ (Theorem 5.4). Consequently if $F_{\sigma}=t_{0} F_{0} t_{\sigma}$, then $a F_{\sigma}=F_{\sigma}$ for all $a \in \sigma$ and $\left|F_{\sigma}\right|=n$. By Theorem 5.4, $S$ is $m$-ELA for some $m \leqq n$. Furthermore $\left|F_{0} s\right|=n$ for all $s \in S$, since $a s=b s, a, b \in F_{0}$ and $s \in S$, then $a s c=b s c$ for some $c \in S$ such that $s c \in T$, which is impossible. Hence $m=n$ (Theorem 5.2). Conversely, if $S$ is $n$-ELA and $F_{0}$ is a coset representative of $S /(r)$, we may assume $F_{0} \subseteq T$ (for otherwise replace $F_{0}$ by $F_{0} s_{0}$, where $s_{0} \in S$ such that $F_{0} s_{0} \subseteq T$ ). If $s_{\sigma}, \mu_{\sigma} \in S$ such that $a F_{0} s_{\sigma}$ $=F_{0} s_{\sigma}$ and $t_{\sigma}=s_{\sigma} \mu_{\sigma} \in T$, then $a F_{0} t_{\sigma}=F_{0} t_{\sigma}$ for all $a \in \sigma$ (Theorem 5.4). Hence $S$ is $m$-ELA for some $m \leqq n$ (Theorem 5.4). Since $\left|F_{0} t\right|=n$ for all $t \in T, m=n$ (Theorem 5.2).

With an observation that every left ideal of a semigroup and every right ideal of a left amenable semigroup is left thick, we obtain the following generalisation of Proposition 7 of Granirer [4] and a remark on p. 113 of [5]: 
COROllary. For any semigroup $S$ :

(a) If $I$ is a left ideal of $S$, then $S$ is $n$-ELA iff I is $n-E L A$.

(b) If $S$ is $n-E L A$, then every right ideal I of $S$ is $n-E L A$.

Remark. Note that the converse of (b) is false even for $n=1$ (see [5, p. 113]).

It has been proved by Day [1, Theorem 2] that if $S$ is left amenable, and $S_{0}$ is a subsemigroup of $S$ such that $\varphi\left(1_{S_{0}}\right)>0$ for some LIM $\varphi$ on $m(S)$, then $S_{0}$ is left amenable. The following proposition, which generalises [4, Proposition 3], is an analogue of this result.

Proposition 5.8. Let $S$ be an $n$-ELA semigroup, and $S_{0}$ a subsemigroup of $S$. If there exists a mean $\varphi_{0}$ on $S$ such that $\varphi_{0}\left(1_{S_{0}}\right)=\varphi_{0}\left({ }_{t} 1_{S_{0}}\right)>0$ for all $t \in S$, then $S_{0}$ is $m-E L A$ for some $m \leqq n$.

Proof. If $\varphi$ is a LIM on $m(S)$, then $\mu=\varphi \odot \varphi_{0}$ is also a LIM on $m(S)$ and $\mu\left(1_{S_{0}}\right)>0$. Let $F$ be a coset representative of $S /(r)$ such that $F \cap S_{0} \neq \varnothing$. If $\sigma$ is a finite subset of $S_{0}, t_{\sigma} \in S$ such that $a F t_{\sigma}=F t_{\sigma}$ for all $a \in \sigma$ (Theorem 5.4) and $s_{\sigma} \in$ $t_{\sigma} S \cap S_{0}$ (which is nonempty, since $\mu\left(1_{t_{\sigma}}\right)=1$ ), then $F_{\sigma}=F s_{\sigma} \cap S_{0} \neq \varnothing$ and $a F_{\sigma}=F_{\sigma}$ for all $a \in \sigma$. Consequently, $S$ is $m$-ELA for some $m \leqq n$ (Remark 5.3).

Granirer [4, Proposition 1a] has proved that any countable subsemigroup of an ELA semigroup $S$ can be embedded in a countable ELA subsemigroup of $S$. His proof can be adopted, with easy modification, to yield the following result; we omit the proof.

Proposition 5.9. If $S$ is an $n$-ELA semigroup, then every countable subsemigroup of $S$ can be embedded in a countable $n$-ELA subsemigroup of $S$.

6. Direct products. We list in this section some basic combinatorial properties of topological semigroups $S$ for which $\operatorname{LUC}(S)$ is $n$-ELA. For analogue results in discrete amenable groups, locally compact amenable groups and ELA discrete semigroups, we refer the reader to Day [1], Rickert [18] and Granirer [4].

Proposition 6.1. Let $S$ and $T$ be topological semigroups, $\pi$ a continuous homomorphisms of $S$ onto $T$. If $L U C(S)$ is $n-E L A$, then $L U C(T)$ is $m-E L A$ for some $m \leqq n, m$ divides $n$.

Proof. If $f \in \operatorname{LUC}(S)$, then $f \circ \pi \in \operatorname{LUC}(S)$. Let $\mu=(1 / n) \sum_{1}^{n} \varphi_{i}, \varphi_{i} \in \Delta(S)$ be a $\operatorname{LIM}$ on $\operatorname{LUC}(S)$, then $\bar{\mu}=(1 / n) \sum_{1}^{n} \bar{\varphi}_{i}$ where $\bar{\varphi}_{i}(f)=\varphi_{i}(f \circ \pi)$ for $f \in \operatorname{LUC}(T)$ is a LIM on LUC(T). The result now follows from Lemma 4.7.

Proposition 6.2. Let $S$ be a topological semigroup, $\left\{S_{\alpha} ; \alpha \in I\right\}$ subsemigroups of $S$ with the induced topology such that $\bigcup_{\alpha \in I} S_{\alpha}=S$ and for each $\alpha, \beta \in I$, there exists $\gamma \in I$ such that $S_{\gamma} \supseteq S_{\alpha} \cup S_{\beta}$. If for each $\alpha \in I, L U C\left(S_{\alpha}\right)$ is $k_{\alpha}$-ELA for some $k_{\alpha} \leqq n$, $k_{\alpha}$ divides $n$, then $L U C(S)$ is $k$-ELA for some $k \leqq n, k$ divides $n$.

Proof. Partially order $I$ by $\alpha \geqq \beta$ iff $S_{\alpha} \supseteq S_{\beta}$. $\geqq$ renders $I$ into a directed set. For $f \in \operatorname{LUC}(S), \alpha \in I$, define $\left(P_{\alpha} f\right)(s)=f(s)$ if $s \in S_{\alpha}$. One readily checks that $\left(P_{\alpha} f\right)$ 
$\in \operatorname{LUC}\left(S_{\alpha}\right)$ and ${ }_{s}\left(P_{\alpha} f\right)=P_{\alpha}(s f)$ for $s \in S_{\alpha}$. For $\alpha \in I$, let $\mu_{\alpha}=(1 / n) \sum_{1}^{n} \varphi_{i}^{\alpha}$ be a LIM on $\operatorname{LUC}\left(S_{\alpha}\right), \varphi_{i}^{\alpha} \in \Delta\left(S_{\alpha}\right)$ ( $\varphi_{i}^{\alpha}$ is not necessarily distinct). Define $\bar{\mu}_{\alpha}=(1 / n) \sum_{1}^{n} \bar{\varphi}_{i}^{\alpha}$ where $\bar{\varphi}_{i}^{\alpha}(f)=\varphi_{i}^{\alpha}\left(P_{\alpha} f\right), f \in \operatorname{LUC}(S)$. By compactness of $\Delta(S)$ and taking subnets if necessary, we may assume $w^{*}-\lim _{\alpha} \bar{\varphi}_{i}^{\alpha}=\psi_{i}$ for some $\psi_{i} \in \Delta(S)$ and each $i=1, \ldots, n$. Then $(1 / n) \sum_{1}^{n} \psi_{i}$ is a $\operatorname{LIM}$ on $\operatorname{LUC}(S)$ since if $s \in S_{\alpha_{0}}, L_{s} \bar{\mu}_{\alpha}=\bar{\mu}_{\alpha}$ for all $\alpha \geqq \alpha_{0}$. Now apply Lemma 4.7 .

For any semigroups $S$ and $T, \varphi \in m(S)^{*}$ and $\mu \in m(T)^{*}$, define $\varphi \times \mu \in m(S \times T)^{*}$ by $(\varphi \times \mu)(f)=\mu(h)$ where $h(t)=\varphi\left(\pi_{t} f\right), f \in m(S),\left(\pi_{t} f\right)(s)=f(s, t)$ for all $s \in S$, $t \in T$.

It is easy to see that if $\varphi$ and $\mu$ are means on $m(S)$ and $m(T)$ respectively, then $\varphi \times \mu$ is a mean on $m(S \times T)$. Furthermore, if $\varphi \in \beta S$, and $\mu \in \beta T$, then $\varphi \times \mu \in$ $\beta(S \times T)$.

LEMMA 6.3. For any topological semigroups $S, T, a \in S, b \in T$ and $f \in L U C(S \times T)$ :

(a) If $t_{0} \in T$, then $\pi_{b t_{0}}(f) \in L U C(S)$.

(b) If $\varphi$ is a mean on $m(S), h(t)=\varphi\left({ }_{a} \pi_{t}(f)\right)$, then $h_{b} \in L U C(T)$.

Proof. (a) Let $\mathscr{E}>0, s_{0} \in S$. Choose $U, V$ neighborhoods of $s_{0}, b$ respectively such that $\left\|_{(u, v)} f-{ }_{\left(s_{0}, b\right)} f\right\|<\mathscr{E}$ for all $(u, v) \in U \times V$. Then for all $u \in U$

$$
\left\|_{u}\left(\pi_{b t_{0}}(f)\right)-_{s_{0}}\left(\pi_{b t_{0}}(f)\right)\right\| \leqq\left\|_{(u, b)} f-{ }_{\left(s_{0}, b\right)} f\right\|<\mathscr{E} .
$$

(b) Let $\mathscr{E}>0, t_{0} \in S, U, V$ be neighborhoods of $a$ and $t_{0}$ respectively such that $\left\|_{(u, v)} f-{ }_{\left(a, t_{0}\right)} f\right\|<\mathscr{E}$ for all $(u, v) \in U \times V$. Then for all $v \in V$

$$
\left\|{ }_{v} h_{b}-{ }_{t_{0}} h_{b}\right\| \leqq\left\|_{(a, v)} f-{ }_{\left(a, t_{0}\right)} f\right\|<\mathscr{E} .
$$

Proposition 6.4. For any topological semigroup $S, T$ and $n$ fixed. $L U C(S \times T)$ is $n$-ELA iff $L U C(S)$ is $k-E L A$ and $L U C(T)$ is $m$-ELA for some $k, m$ such that $k \cdot m=n$.

Proof. Let $\varphi=(1 / k) \sum_{1}^{k} \varphi_{i}$ and $\eta=(1 / m) \sum_{1}^{m} \eta_{j}$ be LIM on LUC $(S)$ and LUC $(T)$ respectively, where $\varphi_{i} \in \Delta(S), \eta_{j} \in \Delta(T)$. If $\tilde{\varphi}_{i} \in \beta S$ and $\tilde{\eta}_{j} \in \beta T$ are multiplicative extensions of $\varphi_{i}$ and $\eta_{j}$ respectively, $\tilde{\varphi}=(1 / k) \sum_{1}^{k} \tilde{\varphi}_{i}, \tilde{\eta}=(1 / m) \sum_{1}^{m} \tilde{\eta}_{j}, \tilde{\psi}=(1 / k) \sum_{1}^{k} \tilde{\psi}_{i}$, where $\tilde{\psi}_{i}=L_{a} \tilde{\varphi}_{i} \in \beta S$ for some fixed $a \in S$, and $\tilde{\gamma}=(1 / m) \sum_{1}^{m} \tilde{\gamma}_{j}$, where $\tilde{\gamma}_{j}(f)=$ $\tilde{\eta}_{,}\left(f_{b}\right)$ for all $f \in m(T)$ and some fixed $b \in T$, we shall show that $\mu=\tilde{\psi} \times \tilde{\gamma}$ when restricted to $\operatorname{LUC}(S \times T)$ is a LIM. Let $\left(s_{0}, t_{0}\right) \in S \times T, f \in \operatorname{LUC}(S \times T)$ be arbitrary but fixed. Define $k(t)=\tilde{\psi}\left(\pi_{t}(f)\right)=\tilde{\varphi}\left({ }_{a} \pi_{t}(f)\right)$. Then $k_{b} \in \operatorname{LUC}(T)$ and for each $t \in T$, $\pi_{t_{0} t} f \in \operatorname{LUC}(S)$ (Lemma 6.3). Hence $\tilde{\psi}\left(\pi_{t}\left({ }_{\left(s_{0}, t_{0}\right)} f\right)\right)=\tilde{\varphi}\left({ }_{s_{0} a} \pi_{t_{0}} t(f)\right)=\tilde{\varphi}\left({ }_{a} \pi_{t_{0} t}(f)\right)={ }_{t_{0}} k(t)$ and $\mu\left({\left(s_{0}, t_{0}\right)}_{f}\right)=\tilde{\eta}\left({ }_{t_{0}} k_{b}\right)=\tilde{\eta}\left(k_{b}\right)=\mu(f)$ since $\tilde{\varphi}$ coincide with $\varphi$ on $\operatorname{LUC}(S)$ and $\tilde{\eta}$ coincide with $\eta$ on $\operatorname{LUC}(T)$. Now since $\mu=(1 / \mathrm{km}) \sum_{i, j=1}^{k, m} \tilde{\psi}_{i} \times \tilde{\gamma}_{j}$, and $\tilde{\psi}_{i} \times \tilde{\gamma}_{j}$ are multiplicative, $\operatorname{LUC}(S \times T)$ is $n$-ELA for some $n \leqq k \cdot m$ (Lemma 4.7).

Let $H_{0}=\left\{\varphi_{1}, \ldots, \varphi_{k}\right\}, F_{0}=\left\{\eta_{1}, \ldots, \eta_{m}\right\}$ and $K_{0}=\left\{\psi_{i} \times \gamma_{j} ; i=1, \ldots, k, j=1, \ldots, m\right\}$, where $\psi_{i} \times \gamma_{j}$ is the restriction of $\tilde{\psi}_{i} \times \tilde{\gamma}_{j}$ to $\operatorname{LUC}(S \times T)$. We shall show that the factor semigroup $(S \times T) / K_{0}$ is isomorphic to $S / H_{0} \times T / F_{0}$ and hence $n=k \cdot m$ by Theorem 4.1. 
If $\left(s_{1}, t_{1}\right),\left(s_{2}, t_{2}\right) \in S \times T$ such that $L_{s_{1}} \varphi_{i}=L_{s_{2}} \varphi_{i}$ and $L_{t_{1}} \eta_{j}=L_{t_{2}} \eta_{j}, 1 \leqq i \leqq k, 1 \leqq j \leqq m$, then $\left(L_{s_{1}} \tilde{\psi}_{i}\right)(f)=\left(L_{s_{2}} \tilde{\psi}_{i}\right)(f)$ and $\left(L_{t_{1}} \tilde{\gamma}_{j}\right)(g)=\left(L_{t_{2}} \tilde{\gamma}_{j}\right)(g)$ for all $1 \leqq i \leqq k, 1 \leqq j \leqq m$, $f \in \operatorname{LUC}(S)$ and $g \in \operatorname{LUC}(S)$. Furthermore, for any $\psi_{i} \times \gamma_{j} \in K_{0}, f \in \operatorname{LUC}(S)$, $h_{1}(t)=\tilde{\psi}_{i}\left(s_{s_{1}} \pi_{t}(f)\right) h_{2}(t)=\tilde{\psi}_{i}\left(s_{2}\left(\pi_{i}(f)\right)\right)$, we have $h_{1_{b}}, h_{2_{b}} \in \operatorname{LUC}(T)$ and $\pi_{t_{1} t}(f) \in \operatorname{LUC}(S)$ for all $t \in T$ (Lemma 6.3). Hence ${ }_{t_{1}} h_{1}(t)=\tilde{\psi}_{i}\left(s_{1} \pi_{t_{1}} t(f)\right)=\tilde{\psi}_{i}\left(s_{2} \pi_{t_{1} t}(f)\right)={ }_{t_{1}} h_{2}(t)$, and

$$
\left(\psi_{i} \times \gamma_{j}\right)\left({\left(s, t, t_{1}\right)}_{1} f\right)=\tilde{\eta}_{j}\left(t_{1} h_{1_{b}}\right)=\tilde{\eta}_{j}\left(t_{2} h_{2_{b}}\right)=\left(\psi_{i} \times \gamma_{j}\right)\left(s_{s_{2}, t_{2}} f\right) .
$$

Consequently, if $\phi:\left(s^{\prime}, t^{\prime}\right) \rightarrow(s, t)^{\prime}$ for all $(s, t) \in S \times T$, where $s^{\prime}, t^{\prime}$ and $(s, t)^{\prime}$ are the homomorphic images of $s, t$ and $(s, t)$ in $S / H_{0}, T / F_{0}$ and $S \times T / K_{0}$ respectively, then $\phi$ is a homomorphism of $S / H_{0} \times T / F_{0}$ onto $S \times T / K_{0}$. Further $\phi$ is oneone, since if $s_{1}, s_{2} \in S, s_{1}^{\prime} \neq s_{2}^{\prime}$, let $\varphi_{i_{0}} \in H_{0}, f \in \operatorname{LUC}(S)$ such that $L_{s_{1}} \varphi_{i_{0}}(f) \neq L_{s_{2}} \varphi_{i_{0}}(f)$. Define $\tilde{f} \in \operatorname{LUC}(S \times T)$ by $\tilde{f}(s, t)=f(s)$ for all $(s, t) \in S \times T$. Then $\pi_{t}(\tilde{f})=f$ for all $t \in T$. Since $(1 / k) \sum_{1}^{k} \tilde{\psi}_{i}$ coincide with $(1 / k) \sum_{1}^{k} \varphi_{i}$ on $\operatorname{LUC}(S)$, there exists $\tilde{\psi}_{i_{v}}$, say, whose restriction to $\operatorname{LUC}(S)$ is $\varphi_{i,}$. Hence

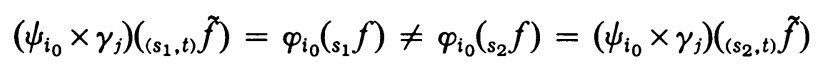

for all $t \in T$ and $1 \leqq j \leqq m$, i.e. $\left(s_{1}, t\right)^{\prime} \neq\left(s_{2}, t\right)^{\prime}$ for all $t \in T$. Similarly, we show that if $t_{1}, t_{2} \in T$ such that $t_{1}^{\prime} \neq t_{2}^{\prime}$, then $\left(s, t_{1}\right)^{\prime} \neq\left(s, t_{2}\right)^{\prime}$ for all $s \in S$. The converse of this proposition follows from Proposition 6.1 and the observation that the mappings $(s, t) \rightarrow s$ and $(s, t) \rightarrow t$ are continuous homomorphisms of $S \times T$ onto $S$ and $T$ respectively.

Let $\left\{S_{\alpha} ; \alpha \in I\right\}$ be semigroups and $\pi_{\alpha \in I} S_{\alpha}$ be the set of all functions defined on $I$ with $f(\alpha) \in S_{\alpha}$ for all $\alpha \in I$. If $f, g \in \pi_{\alpha \in I} S_{\alpha}$, define the product $h=f g$ by $h(\alpha)=$ $f(\alpha) g(\alpha)$ for each $\alpha \in I$. The semigroup $\pi_{\alpha \in I} S_{\alpha}$ is said to be the full direct product of $\left\{S_{\alpha} ; \alpha \in I\right\}$.

If for each $\alpha \in I, S_{\alpha}$ has identity $e_{\alpha}$, let $\pi_{\alpha \in I}^{w} S_{\alpha}$, the weak direct product of $\left\{S_{\alpha}\right.$; $\alpha \in I\}$ be the subsemigroup of all $f \in \pi_{\alpha \in I} S_{\alpha}$ such that $\left\{\alpha \in I ; f(\alpha)=e_{\alpha}\right\}$ is finite. Furthermore, if each $S_{\alpha}$ is a topological semigroup, then $\pi_{\alpha \in I}^{w} S_{\alpha}$ with the pointwise topology is also a topological semigroup.

Proposition 6.5. Let $\left\{S_{\alpha} ; \alpha \in I\right\}$ be topological semigroups with identity, $S=$ $\pi_{\alpha \in I}^{w} S_{\alpha}, n$ fixed. Then LUC $(S)$ is $n-E L A$ iff $L U C\left(S_{\alpha}\right)$ is ELA for all but finitely many $n_{\alpha_{1}}, \ldots, n_{\alpha_{k}}$ for which $1<n_{\alpha_{j}} \leqq n$ and $n=n_{\alpha_{1}} \cdots n_{\alpha_{k}}$.

Proof. If $\operatorname{LUC}\left(S_{\alpha}\right)$ is $n_{\alpha}$-ELA for each $\alpha \in I$, let $F=\{\sigma: \sigma$ finite subset of $I\}$. Order $F$ be upward inclusion. For each $\sigma=\left\{\alpha_{1} \cdots \alpha_{k}\right\} \in F, T_{\sigma}=\left\{f \in S ; f(\alpha)=e_{\alpha}\right.$, the identity of $S_{\alpha}$ for $\left.\alpha \notin \sigma\right\}$ is homeomorphic and isomorphic to $S_{\alpha_{1}} \times \cdots \times S_{\alpha_{k}}$ and hence $\operatorname{LUC}\left(T_{\sigma}\right)$ is $k_{\sigma}$-ELA, where $k_{\sigma}=n_{\alpha_{1}} \cdots n_{\alpha_{k}} \leqq n, k_{\sigma}$ divides $n$ (Proposition 6.4). Furthermore, $\bigcup_{\sigma \in F} T_{\sigma}=S$. Hence $\operatorname{LUC}(S)$ is $m$-ELA for some $m \leqq n$ (Proposition 6.2). Choose $\left\{\alpha_{1} \cdots \alpha_{k}\right\} \subseteq I$ such that $n_{\alpha_{1}} \cdots n_{\alpha_{k}}=n$, then $\operatorname{LUC}\left(S_{\alpha_{1}} \times \cdots \times S_{\alpha_{k}}\right)$ is $n$-ELA, $n \leqq m$, since the mapping $f \rightarrow\left(f\left(\alpha_{1}\right), \ldots, f\left(\alpha_{k}\right)\right)$ is a continuous homomorphism of $S$ onto $S_{\alpha_{1}} \times \cdots \times S_{\alpha_{k}}$. Consequently $n=m$. Conversely, if LUC(S) is $n$-ELA, then as shown above, for each $\alpha \in I, \operatorname{LUC}\left(S_{\alpha}\right)$ is $n_{\alpha}$-ELA, and for each 
finite subset $\sigma=\left\{\alpha_{1} \cdots \alpha_{k}\right\} \subseteq I, \operatorname{LUC}\left(S_{\alpha_{1}} \times \cdots \times S_{\alpha_{k}}\right)$ is $k_{\sigma}$-ELA, where $k_{\sigma}=n_{\alpha_{1}} \cdots n_{\alpha_{k}}$. Consequently, $n_{\alpha}>1$ for only finitely many $\alpha$ 's, and if $m=$ product $\left\{n_{\alpha} ; \alpha \in I\right\}$ then $\operatorname{LUC}(S)$ is $m$-ELA by what we have proved. Hence $m=n$.

REMARK. Day has given an example [1, p. 517] to show the full direct product of left amenable semigroups need not be left amenable. However, it has been shown by Granirer [4, Proposition 2] that the full direct product of ELA discrete semigroups is ELA. This leads us to consider the following problem: let $\left\{S_{\alpha}: \alpha \in I\right\}$ be topological semigroups, $S=\pi_{\alpha \in I} S_{\alpha}$ with the product topology. It is easy to see that if $\operatorname{LUC}(S)$ is $n$-ELA, then $\left(^{*}\right) \operatorname{LUC}\left(S_{\alpha}\right)$ is $n_{\alpha}$-ELA for some $n_{\alpha} \leqq n, n_{\alpha}>1$ for finitely many $\alpha$ 's and $n=$ product $\left\{n_{\alpha} ; \alpha \in I\right\}$, since for any finite subset $\sigma=\left\{\alpha_{1} \cdots \alpha_{k}\right\} \subseteq I$, the mapping $f \rightarrow\left(f\left(\alpha_{1}\right), \ldots, f\left(\alpha_{k}\right)\right)$ is a continuous homomorphism of $S$ onto $S_{\alpha_{1}} \times \cdots \times S_{\alpha_{k}}$. But conversely, if $\left(^{*}\right)$ holds, is $\operatorname{LUC}(S) n$-ELA? We do not have an answer even for $n=1$ except when $I$ is finite (which follows from Proposition 6.4 by induction), or when $S_{\alpha}$ are discrete, for which we are going to prove.

LEMMA 6.6. If $\left\{S_{\alpha}, \alpha \in I\right\}$ are semigroups with f.i.p.r.i., then $S=\pi_{\alpha \in I} S_{\alpha}$ has f.i.p.r.i. and $S /(r)$ is isomorphic to $\pi_{\alpha \in I} T_{\alpha}$, where $T_{\alpha}=S_{\alpha} /(r)$.

Proof. Let $f, g \in S$. For $\alpha \in I$, let $h_{1}(\alpha)$ and $h_{2}(\alpha) \in S_{\alpha}$ such that $f(\alpha) h_{1}(\alpha)=$ $g(\alpha) h_{2}(\alpha)$. Then $f h_{1}=g h_{2}$ and $S$ has f.i.p.r.i. If $f^{\prime}$ and $f^{\prime}(\alpha)$ denote the homomorphic images of $f$ and $f(\alpha)$ in $S$ and $S_{\alpha} /(r)$ respectively, $\alpha \in I$, define a mapping: $\phi\left(f^{\prime}\right)(\alpha)$ $=f(\alpha)^{\prime}$ for all $f \in S$ and $\alpha \in I$. One readily checks that $\phi$ is an isomorphism of $S /(r)$ onto $\pi_{\alpha \in I} T_{\alpha}$.

Lemma 6.6 and Theorem 4.2 together yield:

Proposition 6.7. Let $\left\{S_{\alpha} ; \alpha \in I\right\}$ be semigroups, $S=\pi_{\alpha \in I} S_{\alpha}$ and $n$ fixed. Then $S$ is $n$-ELA iff $S_{\alpha}$ is ELA for all but finitely many $n_{\alpha_{1}}, \ldots, n_{\alpha_{k}}$ for which $1<n_{\alpha_{j}} \leqq n$ and $n=n_{\alpha_{1}} \cdots n_{\alpha_{k}}$.

\section{REFERENCES}

1. M. M. Day, Amenable semi-groups, Illinois J. Math. 1 (1957), 509-544. MR 19, 1067.

2. N. Dunford and J. Schwartz, Linear operators. I: General theory, Interscience, New York, 1958. MR 22 \#8302.

3. E. Granirer, A theorem on amenable semi-groups, Trans. Amer. Math. Soc. 111 (1964), 367-379. MR 29 \#3870.

4. —_ Extremely amenable semi-groups, Math. Scand. 17 (1965), 177-197. MR 33 \#5760.

5. —_, Extremely amenable semi-groups. II, Math. Scand. 20 (1967), 93-113. MR 35 \#3422.

6. - Functional analytic properties of extremely amenable semigroups, Trans. Amer. Math. Soc. 137 (1969), 53-76.

7. - On the range of an invariant mean, Trans. Amer. Math. Soc. 125 (1966), 384-394. MR 34 \#4390.

8. - On the invariant mean on topological semi-groups and on topological groups, Pacific J. Math. 15 (1965), 107-140. MR 35 \#286.

9. E. Granirer and A. Lau, Invariant mean on locally compact groups, Illinois J. Math. (to appear). 
10. F. P. Greenleaf, Invariant means on topological groups and their applications, Van Nostrand, Princeton, N. J., 1969.

11. E. Hewitt, On two problems of Urysohn, Ann. of Math. (2) 47 (1946), 503-509. MR 8, 165.

12. E. S. Ljapin, Semi-groups, Fizmatgiz, Moscow, 1960; English transl., Transl. Math. Monographs, vol. 3, Amer. Math. Soc., Providence, R. I., 1963; rev. ed., 1968. MR 22 \#11054.

13. T. Mitchell, Constant functions and left invariant means on semi-groups, Trans. Amer. Math. Soc. 119 (1965), 244-261. MR 33 \#1743.

14. - Fixed points and multiplicative left invariant means, Trans. Amer. Math. Soc. 122 (1966), 195-202. MR 32 \#7662.

15. - Function algebras, means, and fixed points, Trans. Amer. Math. Soc. 130 (1968), 117-126. MR 36 \#666.

16. - Topological semi-groups and fixed points, (to appear).

17. I. Namioka, On certain actions of semi-groups on L-spaces, Studia Math. 29 (1967), 63-77. MR 36 \#6910.

18. N. W. Rickert, Amenable groups and groups with the fixed point property, Trans. Amer. Math. Soc. 127 (1967), 221-232. MR 36 \#5260.

19. J. Sorenson, Existence of measures that are invariant under a semi-group of transformations, Thesis, Purdue University, Lafayette, Ind., 1966.

UNIVERSITY OF BRITISH COLUMBIA, VANCOUVER, B.C., CANADA 